\title{
Impacto socio-económico, político y ambiental de los asentamientos humanos en la periferia de la ciudad.
}

\section{Socio-economic, political and environmental impact of human settlements on the periphery of the city.}

\author{
Luisa Stella Paz Montes ${ }^{1}$, Gloria Nury Chacon ${ }^{2}$, Eduardo Solano Becerra ${ }^{3}$ \\ 1,2,3 Universidad Francisco de Paula Santander, Cúcuta - Colombia \\ ORCID: ${ }^{1}$ 0000-0002-8887-3441, ${ }^{2} \underline{0000-0002-8428-8683,}{ }^{3} \underline{0000-0002-6702-1047}$
}

Recibido: 19 de enero de 2021.

Aceptado: 14 de abril de 2021.

Publicado: 01 de mayo de 2021.

\begin{abstract}
Resumen - La investigación proporciona los resultados del Impacto socioeconómico, político y ambiental de los asentamientos humanos en la periferia de la ciudad de Cúcuta. La investigación tuvo un enfoque mixto, porque se utilizó el método descriptivo y el estudio de campo, permitiendo obtener información primaria de los sujetos mediante encuestas estructuradas aplicadas en la localidad respectiva y consulta a fuentes secundarias. La población corresponde a las familias que están ubicadas en la periferia de la ciudad de Cúcuta, se utilizó el muestreo no probabilístico bajo la técnica de muestreo aleatorio, permitiendo seleccionar 200 familias que cumplen las características y condiciones del estudio. Se evidenció una comunidad con características vulnerables generalizada por falencias de estructuración de vivienda y alto índice de asentamiento humando trayendo como consecuencia abandono o deficiente atención por parte del Estado, la ausencia total de servicios públicos como saneamiento básico, debido a las precarias condiciones en las que habitan la inmensa mayoría de los individuos que allí residen. La totalidad de los núcleos familiares están conformados por madres solteras cabezas de familia con uno o tres hijos, que se desempeñan como trabajadoras independientes, su nivel de educación es básico y le permite trabajar en empleos de oficios varios. El servicio de salud, es deficiente, no existe seguridad social integral. Por último, se constató que los miembros de las familias no cuentan con las condiciones habitacionales adecuadas, ya que la gran mayoría de estos poseen un solo cuarto donde realizan diversas actividades.
\end{abstract}

Palabras clave: Impactos, socioeconómico, político, ambiental, asentamiento humano.

\begin{abstract}
The research provides the results of the socio-economic, political and environmental impact of human settlements on the periphery of the city of Cúcuta. The research had a mixed approach, because the descriptive method and the field study were used, allowing to obtain primary information of the subjects through structured surveys applied in the respective locality and consultation with secondary sources. The population corresponds to the families that are located in the periphery of the city of Cúcuta, non-probabilistic sampling was used under the random sampling technique, allowing to select 200 families that meet the characteristics and conditions of the study. It was evidenced a community with vulnerable characteristics generalized by shortcomings of housing structuring and high settlement rate, resulting in abandonment or poor attention by the State, the total absence of public services as basic sanitation, due to the precarious conditions in which the vast majority of the individuals who live there inhabit. All of the family nuclei are made up of single mothers, heads of households with one or three children, who work as independent workers, their level of education is basic and allows them to work in various jobs. The health service is poor, there is no comprehensive social security. Finally, it was found that family members do not have adequate housing conditions, since the vast majority of these have only one room where they perform various activities.
\end{abstract}

Keywords: impacts, socioeconomic, political, environmental, human settlement. 


\section{INTRODUCCIÓN}

En Colombia muchos de los recursos financieros y aportes en especie que deben ser canalizados hacia las comunidades vulnerables por parte del programa Plan Nacional de Desarrollo y otras entidades gubernamentales que apoyan esta iniciativa, no son distribuidos ni entregados de manera equitativa, generando una fuerte problemática social. Además, otros factores negativos como la violencia han producido que muchas personas dejen sus viviendas y se instalen en diferentes localidades en calidad de desplazados, sufriendo consecuencias como falta de educación, problemas de salud, violencia intrafamiliar entre otros.

Para estudiar el impacto socioeconómico, político y ambiental generado por los asentamientos humanos en la periferia de la Ciudad de Cúcuta, después de una revisión bibliográfica y consulta a profesionales expertos en la temática, se definieron una serie de conceptos y variables intervinientes para indagar sobre el objeto de estudio de la ponencia. Con la realización del diagnóstico se puede conocer el impacto de las diferentes variables que afectan la calidad de vida de la comunidad en mención.

Los asentamientos son comunidades vulnerables que necesita ayuda por parte del Estado Colombiano para lograr un mejor aprovechamiento de los recursos, generando una mejor calidad de vida para aquellas personas que sufren la problemática de insatisfacción de necesidades básicas y legalidad del predio donde se encuentran localizados.

\section{MARCO TEÓRICO}

El fenómeno de formación de asentamientos irregulares no solo debe entenderse como un proceso de ocupación de suelo, sino como una problemática que obedece a causas económicas, estructurales e incluso a conflictos internos y desastres naturales. ONU habitad reconoce el derecho de toda persona a un nivel de vida adecuado, incluida una vivienda apta. A pesar del lugar importante que ocupa este derecho en el sistema jurídico mundial, el número de personas que no cuentan con una vivienda adecuada aumenta cada día más.

Millones de personas en todo el mundo viven en condiciones peligrosas para la vida o la salud, hacinadas en tugurios y asentamientos improvisados, o en otras condiciones que no respetan sus derechos humanos ni su dignidad. Según fuentes del DANE en Colombia el $23.84 \%$ de los hogares habitan en viviendas con problemas de hacinamiento mitigable.

La exclusión social y la desigualdad que se encuentra presente en estas localidades hace vulnerable a los habitantes de este sector, porque se refleja la insatisfacción de las necesidades básicas tales como: vivienda, servicios públicos, salud, educación (analfabetismo), infraestructura, oportunidades laborales; igualmente, se evidencia que la carencia de estas genera asentamiento humano, descomposición en el hogar, desnutrición, enfermedades y un sin número de factores negativos que afectan a la población.

En la actualidad el problema central de este asentamiento humano, consiste en la incertidumbre de un posible desalojo de su localidad debido a que el terreno donde están ubicadas sus viviendas es un predio de propiedad privada, el riesgo de una decisión a favor del propietario y en contra de la comunidad generaría que los habitantes de esta localidad pasarían a integrar las cifras de desplazados interurbanos en la ciudad de San José de Cúcuta.

\section{a. Responsabilidad social - empresarial}

La responsabilidad social corporativa (RSC) también llamada responsabilidad empresarial, se define como la contribución activa y voluntaria al mejoramiento social, económico y ambiental por parte de las empresas, generalmente con el objetivo de mejorar su situación competitiva, valorativa y su valor añadido. En un sentido más general, Correa J (2007) la describe como la responsabilidad que la empresa tiene o asume frente a la sociedad en general.

Mantener la responsabilidad social genera un alto nivel de competitividad y posicionamiento en el mercado, debido a esto la sociedad debe adoptar una serie de políticas que ayuden a los modelos gerenciales a cumplir con los objetivos, políticas como: política ambiental, política de inversión social, política de información y política de cumplimiento de la ley.

Es importante preguntarnos como los expresa Gutierrez R (2006) En medio de un país con grandes desigualdades y azotado por la violencia, ¿cuál es la contribución de los empresarios para construir una sociedad diferente? Para responder esta pregunta se debe examinar qué hacen las empresas colombianas con cada uno de sus grupos de interés, desde los cercanos a su operación hasta aquéllos tan amplios que trascienden a las comunidades aledañas. El aporte y compromiso de los colombianos respecto a la responsabildia social ha venido evolucionando en los últimos años, al pasar de contribiciones caritativas a inversión social y filantropía estratégica.

Actualmente muchas empresas, tales como las cajas de compensación, la andi y Acopi han venido trabajando sobre esta temática y han diseñado estrategias y programas de acción social corporativa; programas de acción social a través de productos y servicios. Tal como lo expresa Atehortúa F (2008) la responsabilidad del empresario ya no es con la organización sino con el entorno, pues la empresa debe generar desarrollo, no solamente económico, sino social, es el compromiso de las empresas de contribuir al desarrollo económico sostenible, trabajando con la comunidad local y la sociedad en general, para mejorar su calidad de vida.

\section{b. Teoría de la transparencia}

Transparencia es un factor clave para generar confianza en la organización y fuera de ella. Cuando los miembros administrativos de una empresa toman decisiones, establecen actividades y asignan recursos de forma oculta, evitando mostrar libremente dichas actividades es lo que se conoce como personas no transparentes, lo cual genera una serie de factores negativos como la desconfianza que debilitan la base y estructura organizacional de dicha compañía. 
Tal como lo escribe el Diario el Comercio (2012) La transparencia siempre ha estado vinculada al principio del control social. Si lo público nos pertenece a todos, entonces cada uno de nosotros tiene el derecho a exigir cuentas sobre ese patrimonio general. No solo eso: cada uno de nosotros tiene la potestad para decidir sobre el uso y destino de dicho patrimonio, al menos en teoría.

En el ámbito internacional, la falta de transparencia se identifica como un problema que afecta la competitividad de las naciones, pues refleja la corrupción que se presenta en las organizaciones tanto públicas como privadas. La transparencia en las organizaciones es un tema relevante, dado que la corrupción es un elemento a considerar en la competitividad de los países y es un reflejo de la forma en que operan las instituciones y empresas.

La transparencia en el entorno organizacional significa que la empresa tiene la disposición de informar sobre su situación actual, de qué decisiones se toman y por quiénes son tomadas (Alonso, 2009). La transparencia resulta ser un factor clave para generar confianza, y esto se logra en la medida en que la empresa responde a las demandas de información sobre la forma de manejarse.

Para Villanueva (2011), la transparencia se refiere a la posición que asumen las organizaciones públicas o privadas de manejarse bajo estándares éticos, de tal forma que los criterios que se siguen, cuando se toma una decisión, puedan presentarse con claridad a las personas que tengan derecho a conocerlos.

La transparencia como categoría ética significa que todas las actividades hechas y dichas se ajusten a una serie de normas. Cuando informamos nos esforzamos en dar a conocer la verdad en base a la normativa correspondiente. Esto debe ser indispensable en el desarrollo de nuestra labor como profesionales dando paso a la confianza y ética global.

\section{c. Teoría de la Confianza}

La confianza es la seguridad que alguien tiene en sí mismo y también el ánimo, el aliento y el vigor para obrar. La confianza es fundamental en una organización, a pesar de que las funciones deben de estar integradas de forma armónica y supervisadas por el administrador o jefe del área, debe brindarles la confianza a todos y cada uno de los trabajadores para que desempeñen las actividades de forma eficiente, eficaz, y por supuesto bien hechas, para que estos se sientan cómodos y autónomos realizando su labor, aumentando de forma positiva la competitividad y producción en el mercado.

\section{METODOLOGÍA O PROCEDIMIENTOS}

El diseño de la investigación "es la estrategia general que adopta el investigador para responder al problema planteado" De castro S., Hoyos M. C. y Umaña. La presente investigación bajo ésta definición se ajusta a las características de una investigación de enfoque mixto (cuantitativo y cualitativo), porque se utiliza el método descriptivo y una técnica de estudio de campo, permitiendo obtener información primaria de los sujetos mediante encuestas estructuradas aplicadas en la localidad respectiva.

La población está conformada por 925 familias que están ubicadas en la periferia de la ciudad de Cúcuta. En la investigación se utilizó el muestreo no probabilístico bajo la técnica de muestreo aleatorio, permitiendo seleccionar 200 familias que cumplen las características y condiciones del estudio.

\section{RESULTADOS ANÁLISIS E INTERPRETACIÓN}

\section{a. Diagnóstico situacional e impacto socio-económico, político y ambiental del asentamiento humano.}

\section{1) Diagnóstico variable datos de la vivienda}

De acuerdo con los resultados de la encuesta, el ochenta por ciento (80\%) de la familias entrevistas, expresan que su vivienda está construida en un predio propio, sin embargo a pesar que cada uno de ellos cancelo un valor por el terreno en donde se encuentra su casa, pero la realidad es que la cancelación del rubro correspondiente fue entregado a un familiar del dueño real pero con desconocimiento de este, porque el legítimo propietario vendió dichos predios a la entidad (Inversiones San José); lo cual representa un conflicto por la propiedad de los terrenos y un posible riesgo de desalojo, que representaría una crisis social y generando posibles manifestaciones por la pérdida de los únicos recursos con los que cuentan los habitantes de este sector, debido a que el nivel de pobreza en el que se encuentran es extremo.

El sesenta y cuatro por ciento (64\%) de las familias encuestadas tienen un material en el piso de sus viviendas de tierra o arena y un cincuenta y nueve por ciento (59\%) del material predominante en las paredes es tabla, burda o tablón, ya que no cuentan con los recursos disponibles para edificar una vivienda con las condiciones más aptas para habitarla, estas circunstancias realmente no son adecuadas para un individuo, puede contraer problemas de salud, en épocas de invierno se puede derrumbar la vivienda, en donde los más afectados por estas precarias condiciones de vida son los menores, debido a su gran exposición a las condiciones ambientales y de inseguridad que priman en cada una de las viviendas y del sector.

Un setenta y dos por ciento ( $72 \%$ ) de las familias encuestadas no cuentan con el servicio de energía eléctrica. El catorce por ciento (14\%) de las familias cuentan con acueducto, el diez por ciento (10\%) con la recolección de basura, tres por ciento (3\%) con alcantarillado y el uno por ciento (1\%) con gas natural; estos resultados dejan claro las duras condiciones de vida a las que se ven enfrentadas todos los días estas familias, teniendo que recurrir a medios poco ortodoxos para poder deshacerse de sus desechos y satisfacer sus necesidades básicas, atentando contra su integridad, su salud, su bienestar.

El asentamiento humano en este sector busca mejora las condiciones de vida de las personas que lo integran; la causa principal para estar ubicados en esta zona, obedece a que las familias que residen en este lugar no cuentan con los recursos financieros disponibles para comprar o 
arrendar una vivienda en una mejor zona de la ciudad de Cúcuta, debido a que sus ingresos mensuales son muy bajos. El treinta y ocho por ciento $(38 \%)$ de las familias son desplazadas, de las cuales el cincuenta y uno por ciento $(51 \%)$ atribuye su desplazamiento a grupos al margen de la ley, lo que obstaculiza severamente su bienestar futuro. Así mismo otro factor que compromete las condiciones de las familias es que muy pocos jefes de hogar se encuentran laborando en empresas que les brindan todos los beneficios y prestaciones legales, que les permita crecer económicamente en el tiempo para mejorar sus condiciones de vida, sin embargo no cuentan con lo básico para satisfacer sus necesidad y aquellas personas que trabajan de forma independiente, realizando diferentes labores, no tienen una estabilidad económica lo que los obliga a buscar los medios que consideren necesarios para sobrevivir ante estas condiciones que se ven enfrentados.

El factor más decisivo para el estado de sus viviendas es la incertidumbre, debido a que no saben con certeza si van a quedarse en estos predios o si por el contrario serán desalojados, razón por la cual no realizan un esfuerzo en tratar de mejorarlas.

\section{2) Diagnóstico variable servicios del hogar}

Los servicios públicos que utilizan son deficientes. En el caso del servicio de alcantarillado, el ochenta y tres por ciento (83\%) de los hogares poseen inodoro conectado a pozo séptico, mientras que el nueve por ciento (9\%), utilizan letrina y el siete por ciento (7\%) manifestó que no tiene servicio sanitario y solamente el restante uno por ciento (1\%) utiliza inodoro conectado al alcantarillado. Con relación al servicio de agua potable para consumo humano, un cincuenta y ocho (58\%) de los hogares obtienen el suministro de agua por tubería que está conectada de manera artesanal; un treinta y tres (33\%) de los hogares recibe el suministro de agua por acueducto; el tres (3\%) de los hogares obtienen el agua potable de la pila pública donde tienen que salir de sus hogares y llevarla en baldes, tobos y demás. El dos (2\%) la obtienen de carro tanque y agua embotellada o en bolsa. Por último, el uno por ciento (1\%) de los hogares recolecta aguas lluvias que es una actividad no recomendable para el consumo humano.

De otra parte, el cuarenta y dos por ciento (42\%) de las familias deben recolectar las basuras y llevarla a otra localidad utilizando los servicios de un operador público o privado; mientras que el $38 \%$ de la comunidad queman o entierran las basuras generando una contaminación ambiental que afecta a los miembros del mismo asentamiento y finalmente un seis por ciento (6\%) deposita las basuras en un patio, lote, zanja o baldío ocasionando malos olores y problemas de salud de las personas que transitan por este sector.

En referencia a la utilización de energía para la cocción de los alimentos en el hogar, el setenta y tres por ciento (73\%) cocina con gas propano en cilindro, el veinte tres por ciento $(23 \%)$ preparan sus alimentos con leña, madera o carbón de leña y tan solo un $4 \%$ con gas natural conectado a red pública.

En general, las condiciones de acceso y utilización de servicios públicos, tales como acueducto, alcantarillado, recolección de basuras y energía en los hogares encuestados es muy deficiente, cabe resaltar que el servicio público de aseo presenta puntos críticos que afectan las condiciones ambientales y sociales del espacio público de la localidad.

\section{3) Diagnóstico variable características y composición del hogar}

En referencia a las características de los miembros del hogar, un cincuenta por ciento (50\%) de las familias de la localidad la integran entre uno y tres miembros (Padre, madre e hijo), debido a que son parejas en busca de formar un hogar para su nueva familia; un cuarenta y ocho por ciento $(48 \%)$ de los hogares están compuestos entre cuatro a seis miembros de familias en unión libre, es decir pocas personas están casadas, viudas o solteras. Así mismo se observó que el setenta y siete por ciento (77\%) de las parejas que conviven en el sector se encuentra entre treinta y uno y cuarenta años de edad y que en el ochenta y dos por ciento (82\%) de los casos el cónyuge vive en el hogar.

Con respecto al nivel de estudios de los padres cabeza de hogar, se evidencio que a medida que la preparación académica aumenta, el numero tanto de padres como de madres que continuaban su formación disminuía, ninguno de los jefes del hogar tiene estudios universitarios, tan solo el uno por ciento (1\%) de estos realizo estudios de educación técnica o tecnológica. Cabe resaltar que el índice de las mujeres es significativamente superior al de los hombres, en la medida que más mujeres terminaron la secundaria y es menor la proporción de los que no realizaron ningún estudio.

La composición de los hogares en referencia al número de hijos, dicho número aumenta de acuerdo con el número de años de convivencia de la pareja, lo que indica que no tienen una planificación familiar, el setenta y tres por ciento (73\%) de los hogares encuestados obtienen los ingresos familiares por medio de actividades independientes que están influenciados por las condiciones cambiantes del entorno económico.

En términos generales, las condiciones económicas de la localidad, las características y capacidades de los miembros del núcleo familiar para obtener ingresos que les permitan tener un nivel de vida aceptable, son insuficientes, a pesar de que reciben ayuda solidaria de un grupo religioso, pero no es suficiente para satisfacer las necesidades básicas.

\section{4) Diagnóstico variable comunidad-relaciones sociales}

El ochenta y siete por ciento de los miembros de la comunidad (87\%) mantienen una relación buena de solidaridad con sus vecinos; un once por ciento $(11 \%)$ mantiene relaciones tensas y difíciles con los vecinos y por ultimo el dos por ciento (2\% mantienen una relación equilibrada con los vecinos en donde se hablan y se colabora lo necesario.

Desde hace algún tiempo el grupo eclesiástico “congregación madre teresa de Calcuta” realiza actividades de integración en esta comunidad, se les contribuye con ayuda en especie (a los niños se les regala ropa, e incluso se realizan donaciones alimentarias)

\section{5) Diagnóstico variable salud}

Con respecto a los resultados del perfil de salud, analizando la afiliación al sistema de salud, tipo de entidad al que se encuentran afiliados y clase de Régimen de Salud, se observó que el $96 \%$ de la población encuestada cuenta con el acceso a algún régimen de salud (Contributivo, 
subsidiado o afiliados a programas de salud pública), pero solo el 14\% de la población encuestada está afiliada a una EPS (en gran medida el pago lo asume el trabajador), no obstante aunque la cifra es baja, esto no representa que este porcentaje de la población cuente con un trabajo formal o cuente con el nivel de ingresos necesarios para cotizar como independiente.

Un factor crítico en la población es el desconocimiento de prácticas saludables que permitan a las personas vivir con mejores condiciones de vida, tales como la eliminación de basuras, residuos, alcantarillado, manipulación de alimentos y consumo de agua purificada.

\section{6) Diagnóstico variable población, mujeres, adolescente, niños y ancianos}

El cuarenta y nueve por ciento (49\%) de las familias tienen entre 1 a 3, considerándose un cantidad normal de hijos para el bienestar de la misma, sin embargo y pese a dichas condiciones el cuarenta y seis por ciento (46\%) de las familias que conforman el sector tienen hijos en un rango entre 4 y 6 , minimizando la posibilidad de vivir de una manera más cómoda debido a que generalmente las casas de este sector son pequeñas y suele ser incomodo el diario vivir, pero aun así logran adaptarse a las condiciones de vida y a los espacios. El cuatro por ciento (4\%) de la población tienen más de 6 hijos lo que se les dificulta aún más la situación económica, por otra parte, se halló que el uno por ciento (1\%) de las parejas no tiene niños pequeños en el hogar, pero sin embargo cuentan con adolescentes.

Con relación al nivel educativo de los hijos, se comprobó que el sesenta y nueve por ciento (69\%) de los niños asisten a la escuela, por lo general las familias que cuentan con una menor cantidad de hijos entre 1 y 3 los envían a la escuela, mientras que aquellas familias que tienen hijos entre 4 y 6 no puede asistir todos, solo uno o dos por falta de recursos económicos. De los niños que hacen parte de esta comunidad desafortunadamente el cuatro por ciento $(4 \%)$ presentan discapacidad, malformaciones al nacer o algún otro tipo de discapacidad, sin embargo y para fortuna de la gran mayoría de familias de este sector el noventa y seis por ciento $(96 \%)$ de niños gozan de buena salud y no cuentan con ninguna discapacidad que afecte su vida.

Un setenta y seis por ciento (76\%) de los jóvenes que integran los hogares encuestados asisten al colegio, en otros casos a la universidad, pero un porcentaje significativo del veinte cuatro por ciento (24\%) no asisten a ningún plantel educativo, por motivos económicos, por falta de interés para alcanzar una mejor calidad de vida o simplemente porque prefieren trabajar para solventar las necesidades por las que se ven afectados en sus hogares.

Particularmente se notó que el sector está altamente poblado por adultos mayores "ancianos" el setenta y nueve por ciento (79\%) de los hogares están conformados por un adulto mayor, el diecisiete por ciento (17\%) por dos adultos mayores y en tan solo el cuatro por ciento (4\%) de la comunidad no hay ancianos en sus hogares.

\section{7) Diagnóstico variable educación}

El catorce por ciento $(14 \%)$ de las personas encuestadas no saben leer ni escribir, predominando en esta categoría las personas de edad avanzada, debido a que no tuvieron la oportunidad de ingresar a una institución educativa en su juventud y en la actualidad no consideran la posibilidad de una preparación, porque para ellos "ya están muy viejos para ingresar a la escuela". Pero esta consideración de los habitantes de no continuar preparándose es un factor común en la mayoría, puesto que el noventa y cinco por ciento (95\%) de los habitantes del sector no asiste a un colegio o universidad, lo que muestra el desinterés por obtener una preparación más avanzada que pudiere darles la oportunidad de mejorar sus condiciones de vida.

Si bien solo el cinco por ciento (5\%) asisten a una institución educativa, se debe tener en cuenta que no cuentan con beneficios que faciliten su estabilidad y condiciones de estudio puesto que el ochenta y siete por ciento (87\%) de los estudiantes no reciben alimentos en las instalaciones, así mismo ningún habitante del sector ha sido beneficiado con una beca para prepararse en alguna institución, por lo cual, solo los que cuenten con los recursos pueden tener acceso a la educación, lo cual se ve reflejado en que tan solo el uno por ciento (1\%) de los habitantes cuenta con el interés y recursos para prepararse a través de un crédito en instituciones.

En general, los habitantes de la localidad presentan dos factores que impiden que tengan acceso a una educación superior, en primer lugar, la mentalidad de que no necesitan estudiar más, puesto que pueden sobrevivir con el trabajo y condiciones actuales que poseen, así como el pensamiento de que ya están demasiado grandes para ingresar a una institución educativa y en segundo lugar y tal vez el aspecto que impacta más a los habitantes es la falta de recursos y oportunidades para acceder a una Institución Educativa, puesto que los recursos que poseen consideran que solo les alcanza para cubrir los gastos del hogar y si incurrieran en estos gastos no tendrían los medios para cubrirlos.

\section{8) Diagnóstico variable fuerza de trabajo}

Se observó un considerado índice de desempleo del cuarenta y nueve por ciento (49\%) incluyendo entre este la dedicación de oficios del hogar realizada por amas de casa, esta situación se considera en la economía como población no activamente productivos dando como resultado una brecha de participación en el mercado laboral contribuyendo a la problemática de vulnerabilidad y pobreza que se percibe en estos hogares. Solo el catorce por ciento (14\%) de estas personas cuentan con algún tipo de contrato por prestación de servicios laborales y el otro $86 \%$ se desempeñan en actividades informales.

\section{9) Diagnóstico variable origen de la población, desplazados e interurbanos}

Al estudiar el origen de la población se encuentra que el treinta y ocho por ciento (38\%) corresponde a personas desplazadas de sus hogares por diferentes problemáticas y esto ha llevado a que se sitúen en este sector con el ánimo de establecerse en un lugar tranquilo, pero sin tener recursos para algo digno. Del número de personas desplazadas, la totalidad proviene de ciudades que enfrentan la realidad del conflicto armado, pobreza y la inseguridad. De gramalote un pueblo que fue destruido por un desastre natural proviene el diez por ciento (10\%) de los desplazados. De Arauca proviene el dieciséis por ciento (16\%) de los desplazados y Tibu dieciocho por ciento 18\%, por conflicto armado.

Varias son las razones que llevaron a estas personas a abandonar sus ciudades. Por grupos al margen de la ley, conflictos armados y violentos 
que hicieron temer por sus vidas corresponde el setenta y ocho por ciento (78\%), es un porcentaje bastante significativo que corresponde a la realidad de nuestro país. Muchas personas se ven perjudicadas por estos conflictos y deciden salir de sus tierras por temor a perder sus vidas. Por causa de extrema pobreza el doce por ciento (12\%) de los desplazados se vio forzados a buscar nuevas alternativas para mitigar este problema, buscando ayuda en otras ciudades.

Estas personas desplazadas, en cuanto a las razones para llegar a Cúcuta, a este sector, se encuentra que un treinta y cinco por ciento (35\%) optaron por venir debido a que poseen familia o conocidos que pudieran bridar un apoyo, el veinte cinco por ciento (25\%) creían encontrar mejores oportunidades de trabajo, un dieciocho por ciento (18\%) de estas personas ven en Cúcuta una ciudad que puede brindar mayor seguridad, de los desplazados de gramalote que fueron reubicados y personas que vinieron por la cercanía de la ciudad provienen un dieciocho por ciento (18\%) .

\section{0) Diagnóstico de la variable condiciones de vida del hogar}

El sector está conformado por casas relativamente pequeñas y estructuras en malas condiciones, sin embargo, allí habitan familias y tienen que emplear un mismo lugar para realizar múltiples funciones. El cuarenta y nueve por ciento (49\%) de las casas están conformadas por un solo cuarto, donde allí mismo cocinan y duermen todos los miembros de la familia, por lo que se hace incómodo y se pueden ver afectado valores personales como la privacidad de las personas, mientras que otro cuarenta y seis por ciento (46\%) de casas cuentan con dos cuartos, donde en uno de ellos duermen hermanos y en el otro padres, por otra parte el cuatro por ciento (4\%) de las casas del sector cuentan con 3 cuartos, son casas en las que los espacios son más fáciles de emplear, y hay un poco más de comodidad en relación con los dos rangos anteriores, tan solo el uno por ciento (1\%) de las casas cuenta con cuatro cuartos.

Para la preparación de los alimentos el treinta y ocho por ciento (38\%) de los hogares realiza esta actividad en un cuarto solo para cocinar, mientras que el cuarenta y uno por ciento $(41 \%)$ de ellos emplean un cuarto para cocinar y dormir. Las familias del sector emplean diferentes lugares para realizar actividades propias de la cocina por ejemplo un siete por ciento (7\%) cocina en la sala y ahí mismo tienen el lavaplatos, mientras que un once por ciento (11\%) cocinan en la sala y lavan sus platos en otro lugar, se encontró además que un dos por ciento (2\%) de esta comunidad, cocina al aire libre en el patio donde allí ubicaron los utensilios de la cocina.

\section{1) Diagnóstico variable capacitación para el trabajo}

Se encontró que en su gran mayoría la población no cuenta con una capacitación que les permita aspirar a un cargo, a través del cual puedan acceder a mejores condiciones de vida.

A su vez, solo el catorce por ciento (14\%) de los jefes de hogar poseen una certificación que los acredita en la práctica de algún trabajo, lo que indica que la inmensa mayoría trabaja en la informalidad, lo cual no les permite ingresar a un mercado laboral que les de beneficios y posibilidades de crecer económicamente a través del tiempo, un aspecto relevante es el desinterés o ignorancia de las personas en la importancia de la preparación y logros académicos, que les permitirá ascender en la búsqueda de mejores oportunidades laborales.

En general, el aspecto crítico que afecta la población es la mentalidad que poseen en cuanto a la necesidad de la preparación y capacitación, puesto que debido a ella no asimilan la importancia que esta tiene para aspirar a conseguir mejores condiciones de vida, que les permita suplir las necesidades del núcleo familiar y asegurar el porvenir de los menores, brindándoles medios a través de los cuales puedan ingresar al mercado laboral con un perfil que les permita ser competitivos.

De igual manera otro aspecto que incide negativamente en las personas es el desconocimiento de los planes y programas que ofrece el gobierno, en el campo de capacitación presencial y virtual de educación técnica y tecnológica, que sin duda mejoraría sus capacidades laborales.

\section{b. Medición de la pobreza}

Tabla 1: Condiciones establecidas para identificación del método de necesidades básicas insatisfechas.

\begin{tabular}{|l|l|}
\hline $\begin{array}{c}\text { NECESIDADES BASICAS } \\
\text { INSATISFECHAS - NBI }\end{array}$ & CONDICIONES \\
\hline VI: vivienda inadecuada & Piso de tierra. \\
\hline VS: vivienda sin servicios & Sin agua por acueducto o sin conexión a alcantarillado o a pozo séptico. \\
\hline HC: hacinamiento critico & Hogares con un número de personas por cuarto superior a tres. \\
\hline DE: dependencia económica & Hogares cuyo jefe tenga un nivel educativo inferior a tercero primaria y tres o más personas por cada persona ocupada. \\
\hline IE: inasistencia escolar & Hogares en los cuales algún niño entre 7 y 11 años. Pariente del jefe, no asista a algún establecimiento educativo. \\
\hline
\end{tabular}

Fuente: Elaboración propia.

Tabla 2: Resultado de la aplicación del método de necesidades básicas insatisfechas

\begin{tabular}{|l|l|}
\hline TIPO DE Necesidades Básicas Insatisfechas NBI & Porcentaje de viviendas \\
\hline Personas una sola NBI & $0.3 \%$ \\
\hline Dos o más NBI & $97.7 \%$ \\
\hline Vivienda inadecuada & $73.7 \%$ \\
\hline Servicios públicos inadecuados & $100 \%$ \\
\hline Hacinamiento critico & $58.6 \%$ \\
\hline Inasistencia escolar & $14.3 \%$ \\
\hline Alta dependencia económica & $71.4 \%$ \\
\hline
\end{tabular}

Fuente: Elaboración propia.

A partir de la aplicación del método de necesidades básicas insatisfechas (NBI) donde se determinaron los resultados del mismo mediante encuestas empleadas a las viviendas del sector, se estableció que los hogares visitados en su gran mayoría presentan más de dos indicadores de NBI con un porcentaje de $97.7 \%$, es decir la localidad presenta una situación de pobreza crónica, para la identificación de dicha conclusión se 


\section{c. Planes gubernamentales que involucran posibles soluciones}

\section{1) Plan nacional de desarrollo}

El propósito del estado es corregir las desigualdades existentes, promover la inclusión y la participación con el fin de garantizar la igualdad y la equidad en los derechos de cada uno de los ciudadanos. Estas bases establecen unos objetivos para la elaboración del Plan, el segundo de ellos lo que busca es integrar las comunidades, contribuyendo al cierre de brechas y tener acceso a bienes públicos y servicios sociales.

Se fundamenta en programas para la educación en menores de edad, como el modelo de Cero a siempre, aseguran el acceso a la salud, educación y vivienda digna para la población, facilitar el acceso a la educación básica media y terciaria ampliando coberturas en los niveles educativos, e implementando las TIC con el objetivo de lograr ser el país más educado de América Latina para el año 2025. Comprometen a las empresas y a gobiernos locales en el apoyo y desarrollo de estrategias que fomenten empleo. Respecto a servicios básicos como energía eléctrica, el gobierno plantea la consolidación de cobertura durante las 24 horas del día, creación de estrategias energéticas e inversión de entidades públicas y privadas con el objetivo que se cuente con este servicio de forma equitativa para todos los colombianos. Por otra parte, el ministerio de minas y energía plantea la prestación de servicio de gas natural, distribuido en redes en diferentes zonas.

La Salud juega un papel importante en las bases para la elaboración del Plan Nacional de Desarrollo, guiándose por la ley 789 de 2002 y definiendo el Sistema de Protección Social con el fin de disminuir la desigualdad en la prestación de este servicio y a mejorar la calidad de vida de los colombianos.

\section{2) Plan departamental de desarrollo}

El Plan de Desarrollo para Norte de Santander es un instrumento de planeación que se presenta como un Plan de Desarrollo Territorial, que al ser concertado por todos los estamentos de la sociedad Norte santandereana se convierte en la guía no sólo del gobierno departamental sino de la ciudadanía en general, con el propósito de alcanzar los resultados de desarrollo contemplados en la Visión Prospectiva 2021.

Los cuales se desagregan en Programas, Subprogramas y Proyectos, que podrán ser monitoreados permanentemente con los informes de gestión y de rendición de cuentas, en donde se podrá hacer seguimiento a las metas de resultado y de productos periódicamente.

\section{3) Plan municipal de desarrollo}

El Plan de Desarrollo Municipal de Cúcuta, recoge la estrategia integral sobre los pilares de familia; generación de empleo; ambiente; alianzas estratégicas nacionales e internacionales hilvanando a través de planes de acción, con la certeza que no solo se está cumpliendo con los cometidos cuatrienales a los que se está obligado por la Ley 152 de 1.994 sino sentando las bases de un horizonte de planeación al 2.033, fecha que se considera icónica como quiera que la ciudad para esa fecha arribará a sus 300 años de fundación.

Adicionalmente, resulta estratégico y fundamental el anclaje que el Plan tiene en los propósitos de los Objetivos del Milenio, expedidos por la Organización de las Naciones Unidas-ONU-; en la estrategia de la red unidos de la Presidencia de la República, en el Plan Nacional de Desarrollo; en la transversalidad de la política de Infancia y adolescencia que se plasma en él; en los postulados de la Agenda de competitividad, y por supuesto en las alianzas estratégicas con el principal aliado, el Gobierno del departamento de Norte de Santander, con quien a través de su Plan desarrollará proyectos conjuntos y hará realidad la necesaria integración en favor de los pueblos.

La pretensión es, entonces, dejar sentadas las bases de lo que será la Cúcuta soñada por todos: la socialmente incluyente y participativa, la urbanísticamente armoniosa, la ambientalmente sostenible, la internacionalmente reconocida, y la institucionalmente sólida y transparente para que las actuales y futuras generaciones, vean reflejadas en ella sus sueños y esperanzas.

\section{CONCLUSIONES}

De acuerdo con los resultados de la encuesta, el origen de la población que integra el asentamiento corresponde al treinta y ocho por ciento (38\%) a personas desplazadas de sus hogares por diferentes problemáticas. Del número de personas desplazadas, la totalidad provienen de ciudades que enfrentan la realidad del conflicto armado, pobreza y la inseguridad en Colombia; De Gramalote un pueblo que fue destruido por un desastre natural proviene el diez por ciento $(10 \%)$ de los desplazados. De Arauca proviene el dieciséis por ciento (16\%) de los desplazados y Tibú dieciocho por ciento $18 \%$, por conflicto armado.

Las familias que integran el asentamiento presentan una grave crisis socioeconómica, debido al decrecimiento del comercio Regional y binacional y la recesión económica que provoca la baja del tipo de cambio de la moneda venezolana, estos factores desembocan a un panorama incierto en cuanto a oportunidades de mejorar la calidad de vida. El factor más decisivo para el estado de sus viviendas es la incertidumbre, debido a que no saben con certeza si van a quedarse en estos predios o si por el contrario serán desalojados, razón por la cual no realizan un esfuerzo en tratar de mejorarlas

Un aspecto crítico que afecta la población es la mentalidad que poseen en cuanto a la necesidad de la preparación y capacitación, puesto que debido a ella no asimilan la importancia que esta tiene para aspirar a conseguir mejores condiciones de vida, que les permita suplir las necesidades del núcleo familiar y asegurar el porvenir de los menores, brindándoles medios a través de los cuales puedan ingresar al mercado laboral; además, las personas desconocen los planes y programas que ofrece el gobierno, en el campo de capacitación presencial y virtual de educación técnica y tecnológica, que sin duda mejoraría sus capacidades laborales. 
De acuerdo con los resultados de la encuesta, el cuarenta y nueve por ciento (49\%) de las familias tienen un número de hijos entre 1 a 3 ; el cuarenta y seis por ciento $(46 \%)$ de las familias que conforman el sector tienen hijos en un rango entre 4 y 6 ; el cuatro por ciento (4\%) de la población tienen más de 6 hijos; el uno por ciento (1\%) de las parejas no tiene niños pequeños en el hogar, pero sin embargo cuentan con adolescentes. El sesenta y nueve por ciento (69\%) de los niños asisten a la escuela, por lo general las familias que cuentan con una menor cantidad de hijos entre 1 y 3 los envían a la escuela, mientras que aquellas familias que tienen hijos entre 4 y 6 no puede asistir todos, solo uno o dos por falta de recursos económicos.

Igualmente, se evidenció que gran parte de los hogares está conformada por mujeres de todas las edades, en dichos núcleos familiares un significativo setenta y nueve por ciento (79\%) cuentan con una y dos mujeres, mientras que el diecisiete por ciento $17 \%$ de los hogares, habitan entre tres y cuatro mujeres. Pocos son los hogares donde no hay mujeres con un seis por ciento (6\%). Por otra parte, el asentamiento está altamente poblado por adultos mayores, el setenta y nueve por ciento (79\%) de los hogares están conformados por un adulto mayor, el diecisiete por ciento (17\%) por dos adultos mayores y en tan solo el cuatro por ciento (4\%) de la comunidad no hay ancianos en sus hogares.

Las condiciones económicas de la localidad, las características y capacidades de los miembros del núcleo familiar para obtener ingresos que les permitan tener un nivel de vida aceptable, son insuficientes, a pesar de que reciben ayuda solidaria de un grupo religioso, pero no es suficiente para satisfacer las necesidades básicas. A partir de la aplicación del método de necesidades básicas insatisfechas (NBI) a los hogares del asentamiento, se estableció que los hogares visitados en su gran mayoría presentan más de dos indicadores de NBI con un porcentaje de $97.7 \%$, es decir la localidad presenta una situación de pobreza crónica, para la identificación de dicha conclusión se realizó la evaluación directa a la población donde denominaron las variables establecidas por el método de NBI

Las condiciones de acceso y utilización de servicios públicos, tales como acueducto, alcantarillado, recolección de basuras y energía en los hogares encuestados es muy deficiente, cabe resaltar que el servicio público de aseo presenta puntos críticos que afectan las condiciones ambientales y sociales del espacio público de la localidad.

Un factor crítico en el asentamiento es el desconocimiento de prácticas saludables que permitan a las personas vivir con mejores condiciones de vida, tales como la eliminación de basuras, residuos, alcantarillado, manipulación de alimentos y consumo de agua purificada.

\section{RECOMENDACIONES}

Es necesario que cada uno de los actores viales piense por un momento en relación a su conducta, hábitos, comportamientos y estilo de conducción, con el objeto de valorar si el mismo puede generar daños materiales o físicos a terceras personas, o en el peor de los casos, puede ser el responsable de la muerte de una persona. Por su parte, se deben integrar las empresas públicas y privadas, los colegios, instituciones educativas y universidades hacia una enseñanza en educación vial, en la que se reconozca los derechos y responsabilidades que le compete al ciudadano, e igualmente se genere el desarrollo de competencias que permitan evaluar los riesgos a los que se está expuesto y tomar las medidas y los comportamientos adecuados en la vía.

\section{REFERENCIAS}

[1] M. Alonso, "La transparencia de las empresas en internet para la confianza de los accionistas e inversoras: Un análisis empírico". 2009 Cuadernos de Administración, 22(38), 11-30.

[2] F. A. Atehortúa-Hurtado "responsabilidad social empresarial: entre la ética discursiva y la racionalidad técnica". Revista Escuela de Administración de Negocios. 2008

[3] J. G. Correa "Evolución histórica de los conceptos de responsabilidad social empresarial y balance social". 2007

[4] S. De castro, M. C. Hoyos y V. Umaña "Legalización de barrios informales Prestación de servicios públicos: ¿una medida constitucional paliativa o un paso hacia la legalización?" (s.f.).

[5] Departamento Nacional De Estadística. DANE. Archivo nacional de datos. 2013.

[6] Departamento Nacional de Planeacion. Versión preliminar para discusión Del consejo nacional de planeación. 2014-2018.

[7] Diario el Comercio. ElComecio.com. https://www.elcomercio.com/opinion/teoria-transparencia.html. 2012

[8] Gobernación de Norte de Santander. Plan de Desarrollo para Norte de Santander. 2012-2015

[9] R. Gutiérrez, L.F Avella y R. Villar. "Aportes y desafíos de la responsabilidad social empresarial en Colombia". Edisoma Ediciones Especiales Ltda. 2006

[10] A. M. Ibáñez, y A. Velásquez "El impacto del desplazamiento forzoso en Colombia: condiciones socioeconómicas de la población desplazada, vinculación a los mercados laborales y políticas públicas”. 2008

[11] Proyecto cátedra UNESCO: derechos humanos y violencia: gobierno y gobernanza. (s.f.). Universidad Externado de Colombia.

[12] Sentencia C-1189/08. Control de constitucionalidad de norma de ley del plan que establece prohibiciones de inversión y servicios públicos en asentamientos ilegales. Publicada en La Sala Plena de la Corte Constitucional.

[13] Sentencia T-1017/07. Derecho a la vivienda digna-Alcance y contenido. Publicada en La Sala Plena de la Corte Constitucional.

[14] Sentencia C-741/03. Prestación de servicios públicos domiciliarios-Regulación legislativa. Publicada en La Sala Plena de la Corte Constitucional.

[15] Sentencia T-309/10. Acción de tutela. Publicada en La Sala Plena de la Corte Constitucional.

[16] Sentencia c-150/03. Referencia: expediente D-4194. Publicada en Gaceta de la Corte Constitucional.

[17] Sentencia C-242/97. Administración de justicia-Función pública. Publicada en La Sala Plena de la Corte Constitucional.

[18] Villanueva, E. Aproximaciones conceptuales a la idea de transparencia. Transparencia: libros, autores e ideas 2011 Disponible en: http://inicio.ifai.org.mx/Publicaciones/tlai18.pdf. 